\title{
Verification in process algebra of the distributed control of track vehicles-A case study*
}

\author{
Siegfried Fischer, Andreas Scholz, Dirk Taubner ${ }^{\dagger}$ \\ Siemens $\Lambda G^{\ddagger}$, Germany
}

\begin{abstract}
A real-life example of parallel processes, namely the distributed control of track vehicles, is modelled and analyzed on design level using process algebra techniques.

We report on the stepwise development, the verification performed, and the errors found. We show how the complexity of verification is handled and argue that the verification was only possible due to computer assistance which was available as the tool PVE (Process Verification Environment). This nontrivial, industrially motivated example may also serve as a benchmark for other methods and tools.
\end{abstract}

\section{Introduction}

Parallel processes more and more often occur in the design of applications such as telecommunicalion systems, operating systems, and embedded control systems. They are extremely hard to test and debug. Opposed to testing, verification aims at proving correctness. A number of approaches such as Petri nets [18], process algebras $[14,1]$, and parallel program verification techniques $[16,5]$ have been developed in order to verify correctness of parallel processes. Examples of applications found in textbooks are typically rather small such as mutual exclusion, dining philosophers, and the alternating bit protocol.

This paper is a case study in applying the techniques of process algebra to a real-life problem. We study the problem of safely controlling track vehicles in a distributed manner. Every track section and every vehicle has its own control processor. These processors have to communicate to ensure safe (e.g. collision-free) movement of vehicles.

This problem is industrially motivated. Such controls are developed in our company and some actual design draft served as the starting point for our study. However due to abstractions, further developments, and for reasons motivated by clarity of presentation the material given in this paper diverges from any actual design.

The aim is to show that the verification techniques supplied by process algebra can be used for practical applications. We want to present a useful approach to designing and verifying a system of parallel processes. We investigate the feasibility and limitations. This case study may also serve as a benchmark for other methods and tools.

It is important to emphasize that we apply verification to the design phase. The reason is twofold. Firstly this cuts short the traditional development cycle where certain errors are found in the test phase only (see Fig. 1). Secondly we believe that only on the design level the system to be verified has a complexity which can be handled with verification. This is true even if stale-of-the-art tool support is used. Nevertheless, due to abstractions some errors will still be detected not earlier than in the test phase.
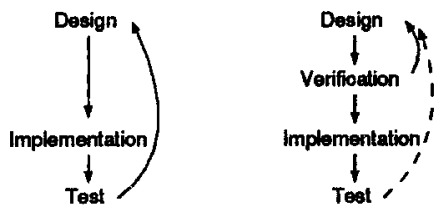

Figure 1: Traditional and refined development cycle

\footnotetext{
"This work has been partially supported by a BMFT grant under contract number TV8743.

iNew addreas: D. Taubner, sd\&m GmbH, Thomas-Deluler-Str. 27, W-8000 München 83, Gernany Email: taubner@informatik.tu-muenchen.de

'Address for correspondence: A. Scholz, Siernens AG, ZFE BT SE 1, Otto-Hahn-Ring 6, W-8000 München 83, Germany Email: scholz@newton.zfe.siemens.de
} 


\section{Process algebra techniques and the tool PVE}

Research on process algebras was initiated by Milner's Calculus of Communicating Systems (CCS) [13], it progressed rapidly, cf. e.g. $[3,8,2,1]$. As we use it, process algebra allows to reason about a system of concurrent finite state machines which communicate synchronously. Two techniques for verification are provided, equivalence checking and model checking. In both cases the external behaviour of the system which is to be verified is relevant.

For equivalence checking one needs a specification (i.e. a second description of this behaviour). The equality of both is then checked with respect to an adequate notion of equivalence. To this end we choose weak bisimulation equivalence, denoled $\approx[14]$.

For model checking one formulates certain desirable properties (such as deadlock freedom, ability to perform certain sequences of actions) in a logic called proposilional modal $\mu$-calculus [9]. Each property is checked for validity against the system. As such properties may cover separate small aspects this approach is feasible even if it is impossible to supply a specificalion for the whole system.

Let us recall how transition systems are combined to a system of communicating components by operators for parallel composition, restriction, and renaming. Assume a set $A c t=\{\tau, a ?, b ?, c ?, \ldots, a !, b !, c !, \ldots\}$ of actions. It contains the internal action $\tau$ which is not visible externally, and as many visible actions as needed. An input action a? will match the output action a! in communications. The basic building block of parallel systems is a transilion system $T=\langle S, \longrightarrow, z\rangle$ which consists of a finite set $S$ of states, a finite ternary transition relation $\longrightarrow \subseteq S \times A c t \times S$, and a starting state $z \in S$. A transition $\langle r, \alpha, s\rangle \in \longrightarrow$ is also denoted as $r-\alpha \rightarrow s$.

The parallel composition $T_{1} \mid T_{2}$ of two transition systems $T_{1}=\left\langle S_{1}, \longrightarrow_{1}, z_{1}\right\rangle$ and $T_{2}=\left\langle S_{2}, \longrightarrow 2, z_{2}\right\rangle$ is defined as $\left\langle S_{1} \times S_{2}, \longrightarrow,\left\langle z_{1}, z_{2}\right\rangle\right)$ where

$$
\longrightarrow:=\quad \begin{aligned}
& \left\{\left\langle\left\langle r_{1}, s_{2}\right\rangle, \alpha,\left(s_{1}, s_{2}\right\rangle\right\rangle \mid r_{1}-\alpha \rightarrow s_{1}\right\} \cup\left\{\left\langle\left(s_{1}, r_{2}\right\rangle, \alpha,\left\langle s_{1}, s_{2}\right\rangle\right) \mid r_{2}-\alpha \rightarrow s_{2}\right\} \\
& \left\{\left\langle\left\langle r_{1}, r_{2}\right\rangle, \tau,\left(s_{1}, s_{2}\right\rangle\right\rangle \mid r_{1}-x ? \rightarrow s_{1} \wedge s_{2}-x ! s_{2} s_{2} \vee r_{1}-x ! \rightarrow_{1} s_{1} \wedge r_{2}-x ? \rightarrow s_{2} s_{2}\right\} .
\end{aligned}
$$

The first two cases allow components to proceed independently asynchronously while the third case constitutes a synchronous communication via matching input and output actions. The joint aclion resulting from the communication is invisible. This is the parallel composition of CCS [14], for others see [20]. Note that it allows only binary synchronization, i.e. the result of a synchronization is invisible and lence carnot serve for an additional synclironization.

To enforce communication one has to disallow the asynchronous moves with the following restriction operator. Given $T=\langle S, \rightarrow, z\rangle$ and a sel $A$ of visible actions, the restriction $T \backslash A$ is defined as $\langle S,\{\langle r, \alpha, s\rangle \mid r-\alpha \rightarrow s \wedge \alpha \notin A\}, z\rangle$. The restriction disallows all transitions labelled by actions in $A$. We often use the complementary restriction operator $T$ allowonly $A:=T \backslash(A c t-(A \cup\{\tau\}))$.

The renaming operator is convenient to instantiate a copy of a transition system with modified actions. Let $f: A c t \rightarrow A c t$ be a function which does not change the invisible action, then $T$ map $f$ is defined as $\langle S,\{\langle r, f(\alpha), s\rangle \mid r-\alpha \rightarrow s\}, z\rangle$.

The tool PVE (Proeess Verification Environment) We have built the software tool PVE [11] which automates process algebra techniques. See $[11,12]$ for overviews over similar tools. In essence PVI provides the basic functionality of the Concurrency Workbench [6] but is much more efficient in space and time. PVE supports the following points.

Parsing A machine-readable syntax for basic transition systems (adapted from [14]), for the process algebra operators, for formulas of the used modal logic, and for several commands has been defined. It is analyzed in a commasd line interpreler which initiales the appropriate functions.

Transition system generation For process algebra source code the transition systems are built internally as an appropriale linked data structure. The $\tau$-closure, i.e. the $\Longrightarrow$ transition relation [11] is computed.

Equivalence checking and Minimization The partition refinement algorillim of Paige and Tarjan [17] has been generalized for the case that there is more than one action. We perform some equivalencepreserving reduction of transition systems before equivalence checking. In addition, the generalized Paige/Tarjan-algorilhnı is used for minimization of transition systems.

Model checking The model checker of Emerson and Lei [9] has been implemented. On an experimental basis we also implemented symbolic model checking $[10,4]$ and local model checking [19]. We plan to integrate the improvements of [7].

PVE has been implemented in C++ and runs on UNIX workstations (HP/Apollo, Sun SPARCstation 2).

Currently the system is able to handle up to $10^{6}$ states and transitions. 


\section{Distributed control technique for track vehicles}

Problem description As is usual for the control of track vehicles, such as railway trains, the track is divided into sections. One such section is called a block. The point which is new here is that the track system is nol controlled centrally (either by hand or machine), but in a decentralized manner. Each block has its own control computer which runs sone software. Taking hardware and software together, we speak of a block control. In addition, each vehicle has its own control computer. The problem of our case study is to design and verify a distributed block controt.

The most important safety requirement is that each block must not contain more than one vehicle (i.e. mutual exclusion is guaranteed). As a vehicle has spatial extension a vehicle may be in two blocks at the same time, cf. Fig. 2. However, we assume a reference point for each vehicle. The transition of this point from one block to the next is assumed to be atomic. A second important property of the control mechanism which we want to verify is the absence of deadlocks. Although a deadlock would not imply a safety risk such as a collision, we do not want to see passengers starving on the track.

All block controls and vehicles have to communicate in order to ensure the correct function of the system. To this end the block controls are linked according to the topologic structure of the track system. In this paper we consider only cyclic topologies. Conmunication between a vehicle and a block control is possible if and only if the reference point of the vehicle is within the block. In this case we say that the vehicle is controlled by the block control. Fig. 2 summarizes the communication structure.

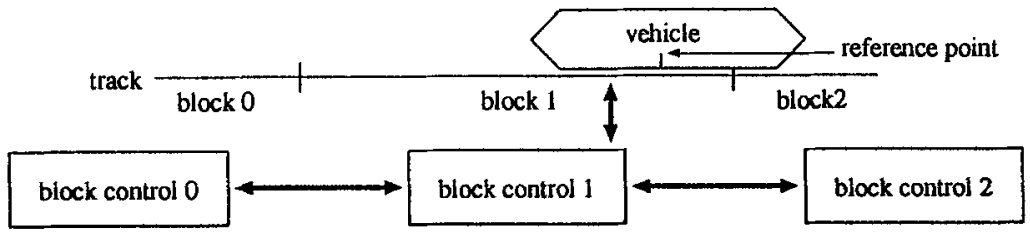

Figure 2: Communication between block controls and a vehicle

The aim of this paper is to present the development process of the design of the block control. We proceed in two steps, first the unidirectional case is considered, later changes of direction are supported additionally. The control system will allow changes of direction at any moment, i.e. the vehicles may attempt to change direction at any position in or "between" blocks.

Modelling of velicles Developing the block control we will need some assumptions on the vehicles and their ability to communicate. In particular we assume that a vehicle which is controlled by a block control informs the control about all important changes of state. We will use the following actions.

Enter The reference point of the vehicle enters the block.

EnterComp The velicle has completely entered the block, no other block is occupied anymore, We assume that blocks are much longer than vehicles.

ResReq The vehicle requests the reservation of the successor block as it approaches the block boundary.

OccSucc The velucle has (partially) occupied the successor block. The distributed block control has to be designed in such a way that this action can be performed only if the successor block is not occupied by another vehicle. Of course automatic braking should start if this action cannot be performed in time. However, this mechanism is not modelled here.

ChDir The vehicle changes its direction of movement.

ChDirOk The vehicle receives a message that the change of direction bas been processed by the control.

Exit The reference point of the vehicle exits the block.

In essence these actions are an abstract model of a track vehicle and its physical movement.

Naming Couventions For the rest of this paper we use a structure for actions which shows the participants in a communication. Every action is prefixed by two characters which indicate the sender and the receiver respectively. For example the aclion VIEnterComp means that vehicle $V$ sends an EnterCompmessage to block control $I$. We will use the following letters in prefixes. 
$V$ Vehicle

I the currently considered block control; may be read as I=myself

$P$ Predecessor of the current block control with respect to the current direction of movement of the vehicle (if there is any in the block)

$S$ Successor of the current block, note that there are only cyclic topologies, so there is exactly one successor

$L \quad$ Left neighbour of the current block with respect to the physical topology

$R$ Right neighbour

$X$ any

Note that these abbreviations are understood with respect to a specific block control. Consider, for example, the complete unidirectional system with movement fron lower to higher block numbers. It is built from several instantialions of the block control such that an action ISxxx of block control 2 matches action $P I x x x$ of block control 3. In process algebra we map both to $23 x x x$ replacing $I, P$ and $S$ by the corresponding block numbers. In diagrams of transition systems we use the additional convention to omit the '?' and '!' marks if they are clear from the two-character prefix. For example in the transition system of the block control I all actions IXxxx (XIXxx) implicitly have an '!' ('?') at the end.

\section{Designing the distributed block control}

This section describes the design process for the block control associated with each block of the track. In a first step we develop a version which is able to handle vehicles that never change their direction of movement. In 4.2 we refine the layout developed in 4.1 and the velicles are allowed to change their direction of movement. at any time.

\subsection{Unidirectional vehicles}

The unidirectional case of our block control will only be able to control vehicles passing from left to right (or in the opposite direction) through a block, but never allows a change of direction. Consequently we speak of predecessor blocks and of successor blocks with respect to a fixed direction of movement for the rest of 4.1. We chose the direction from lower to higher numbers (modulo cycle length).

Specification One possibility for the specification would be to describe the behaviour of a single-block control. However, this is not realistic if nothing is known about the vehicle. Therefore, we start to assume that a vehicle performs the following sequence of actions when moving from block $I$ to block $S$.

\section{VIResReq!.VIOccSucc!.VEinBS!.VIExit!.VSEnter!.VSEnterComp!}

The action VEinBS is to indicate externally (for verification purposes) that vehicle $V$ is starting to move to block $S$. We start with a system of three blocks connected in a cycle. The transition system VehicleUD, given in Fig. 3 shows a vehicle which starts in block number $i$ and which tries to move circularly in one direction through this ring.

The design of the block control has to be such that if we take one copy for each block and link the communication appropriately the vehicle is conlrolled in such a way that it actually can move in circles. In other words if we enforce communication with respect to all actions between block controls and the vehicle we expect the behaviour of the complete system to allow forever the sequence

$$
\text { VEinB1! VEInB2! VEinB0! ... }
$$

To put it formally, if $B C 0 w V, B C 1$, and $B C 2$ are block controls such that $B C O w V$ controls the vehicle initially we expect

$$
(B C 0 w V|B C 1| B C 2 \mid \text { VehicleUD } 0) \text { allowonly \{VEinB0!, VEinB1!, VEinB2!\} }
$$

to be bisimulation equivalent to the transition system of Fig. 4(a). We call this transition system SpecUD1. It is the specification for a unidirectional ring of three blocks with one vehicle.

The specification for a ring which has two vehicles is more interesting. In principle each vehicle can be in one of the three blocks. This would result in 9 possible states. However, those combinations where both vehicles are in the same blcok have to be excluded. The transition system SpecUD2 for the specification of a ring with two vehicles which both move in only one and the same direction is given in Fig. 4(b).

We are now ready for the design. 


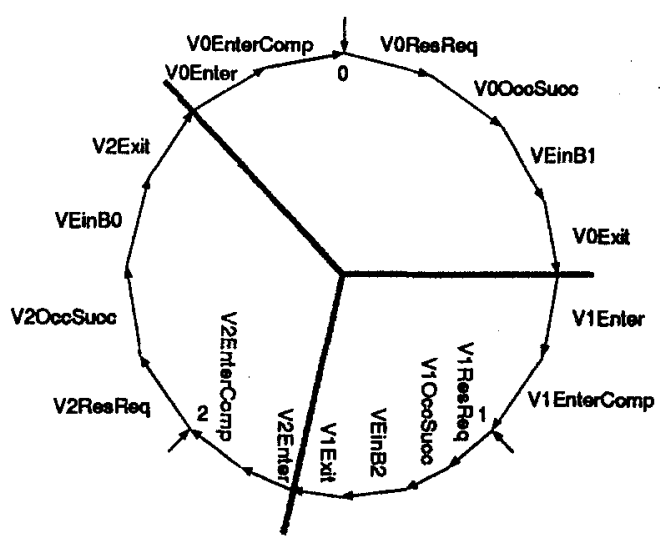

Figure 3: A unidirectional vehicle for a ring of three blocks

(a)

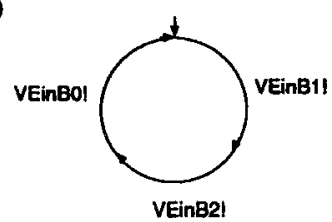

(b)

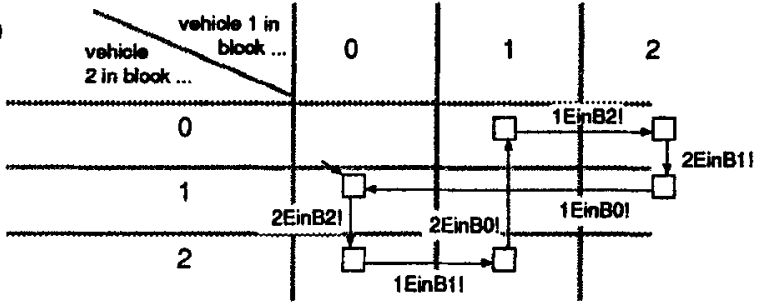

Figure 4: Specification for a unidirectional ring of three blocks with one (a) or two (b) vehicle(s)

Internal control As mutual exclusion on occupation of blocks is the main safety property which is to be ensured by the block control a semaphore is naturally chosen for the beginning of the design process. However, the control of a block is not informed directly by the vehicle about its entering. This is due to the assumption that vehicles communicate only with that block control whose block contains its reference point (see Fig. 2). Therefore, the semaphore has to be enhanced by a reservation phase which allows a vehicle to occupy the block without direct notification of the corresponding block control. In a symmetric way we add a release stale for exiting vehicles. Fig. 5 shows the resulting 4-state process. We will refer to this process as internal control process (IC-process) and to its states as ICx where $x$ is a state uumber.

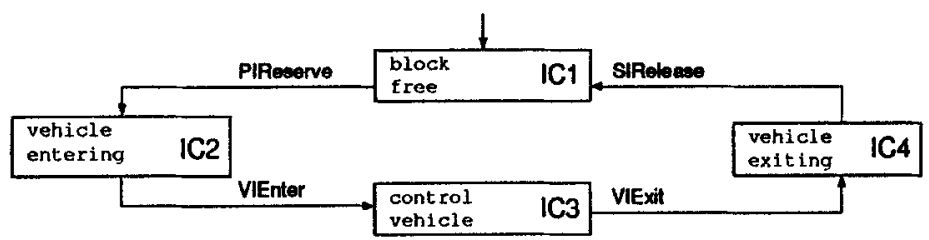

Figure 5: A semaphore enhanced by reservation

Predecessor and successor control As there is a reservation required before occupation there must be someone to make it. For the reasons mentioned above the vehicle cannot do that itselr. We decided to add a second process for this task which is called successor control process (SC-process). It has to perform two subtasks. Firstly it has to perform the reservation procedure upon the receipt of a VIResReq-message. Secondly the process has to ensure that a VIOccSucc-action cannot occur before the reservation is finished successfully. Fig. 6(a) shows the transition diagram for the SC-process. 

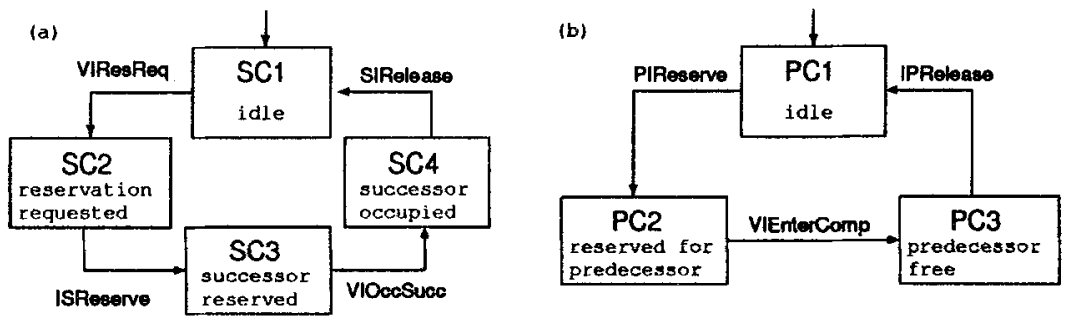

Figure 6: The successor (a) and predecessor (b) control processes

Note that an occupation of the successor block can occur only after a successful reservation. If the successor block cannol be reserved (i.e. the process is blocked in state SC2), the vehicle will be unable to perform a VIOccSucc-action and will be forced to stop by some sort of timeout mechanism within the vehicle which is not modelled in Fig. 3.

A further task which has to be carried out by the block control is to release the predecessor block if the vehicle has completely entered the block. Therefore we add a third process named predecessor control process (PC-process). It has roughly the layout of a boolean variable which indicates whether the predecessor block is occupied by (a part of) a vehicle. Fig. 6(b) shows the transition system of the process. State PC3 can be interpreted as a one-message buffer for the message coming from the vehicle.

Multiple synchronizations Note that the action IPRelease of the PC-process has to match the actions named SIRelease of the internal and the successor control processes of the predecessor's block control. This is an example for a synchronization of more than two processes which is called a multiple synchronizalion. Another example is the XXReserve-action.

The CCS-parallel composition presented in Section 2 allows to express binary synchronizations only. One possibility to overcome this restriction would be to use more powerful synchronizing constructs as proposed e.g. in MEIJE [8]. However the use of multiple synchronizations as a primitive raises the question of adequacy for the description of distributed designs and of implementability. We suggest to allow the use of multiple synchronizations only between processes which are located on the same computer. Otherwise we suggest to use only binary synchronizations.

In order not to limit the actual implementation we do not use multiple synchronizations as primitive construct in this paper. This also yields a deeper insight into the necessary synchronizations which is valuable for a later implementation.

For modelling of multi-synchronizations by binary synchronizations we introduce a communication pattern which we will often use below. We explain it with the example of XXReserve. Fig. 7(a) shows a block control $A$ which requests reservation of its successor block $B$. Therefore it has to synchronize its own successor control process with the internal and predecessor control processes of its successor. We split the action ISReserve into two new actions called ISResReq and SIRes $O \mathrm{k}$ which indicate a reservation request and a positive acknowledgement respectively. Careful design ensures that no deadlocking can occur in the new intermediate states shown in Fig. 7(b). This is of course verified using PVE. For internal synchronization another new action called IIReserve is introduced. A similar construction is applied to the action XXRelease, the new actions are called XXRelReq, XXRelOk and IIRelease.

The complete model and its verification Fig. 8 shows the complete three-process structure of one block control. Naming the component processes with their initial stales we have the following definition.

$$
\text { BlockControlUD : }=(P C 1|I C 1| S C 1) \backslash A_{I I}
$$

Where the set $A_{I I}$ contains all actions which start with II. A block control which controls a vehicle initially is defined as

$$
\text { BlockControlUDWith Vel : }=(P C 1|I C 3| S C 1) \backslash A_{I I} \text {. }
$$

Three copies of the block control for the track are instanliated as follows.

$$
\begin{array}{lllll}
\text { BCOWV } & :=\text { BlockControlUDWithVeh } & \text { map } & \text { fPIS201 } \\
B C 1 & :=\text { BlockControlUD } & \text { map } & \text { fPIS012 } \\
\text { BC2 } & := & \text { BlockControlUD } & \text { map } & \text { fPIS120 }
\end{array}
$$




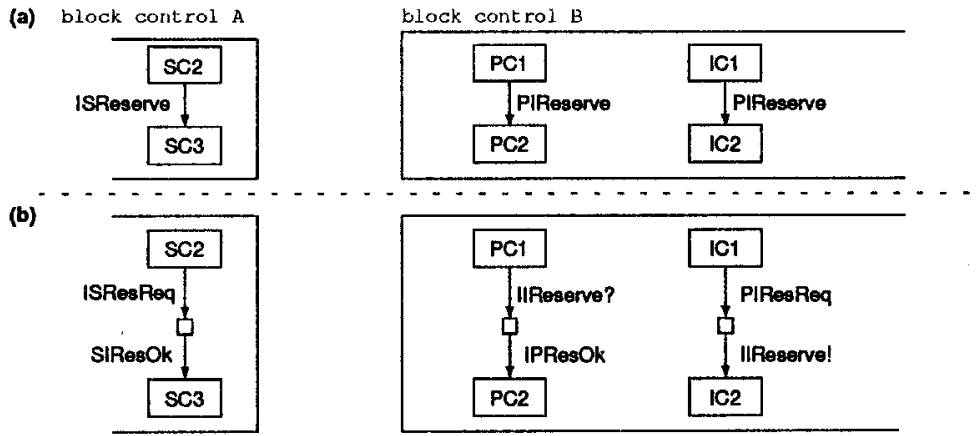

Figure 7: Modelling a multi-synchronization with binary synclironizations
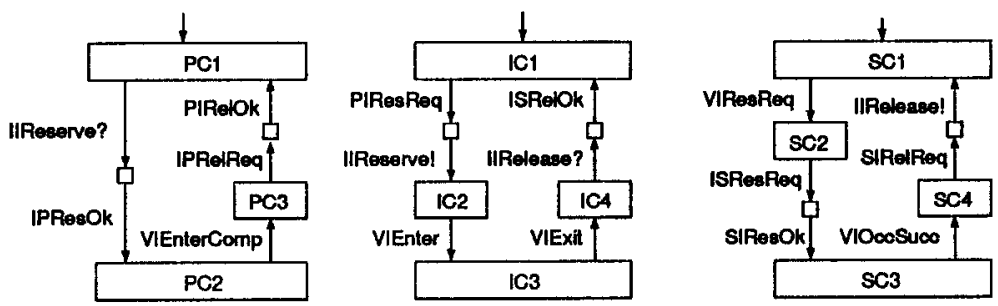

Figure 8: 'The complete block control for unidirectional vehicles

Here fPIS201 maps every $P, I$, and $S$ in the first two characters of actions to 2,0 , and 1 respectively. The functions IPISO12 and fPIS120 are analogous. The complete design of a unidirectional ring of three blocks with one velicle is defined as

$$
\text { RingUD1 := }(B C 0 w V|B C 1| B C 2 \mid \text { VehicleUD })_{0} \text { allowonly } A_{X E}
$$

where $A_{X E}$ contains all actions whose second character is an $E$.

This complete design can now be verified against the specification SpecUD1 of Fig. 4(a). We have performed not only this check with PVE, but also checked several intermediate drafis of the design. We found a number of errors, below in 4.2 we point to a particular error. For the above presented design we have RingUDI $\approx$ SpecUDI.

Similarly for a ring wilh (wo vehicles we instantiate block control 1 as BlockControlUDWilh Veh and two vehicles which are located in block 0 and 1 respectively. Formally we get

$$
\begin{array}{llll}
\text { BC1wV } & :=\text { BlockControlUDWithVeh } & \text { map } & \text { fPIS012 } \\
V 1 & :=\text { VehicleUD } & \text { map } & \text { fVE1E } \\
\text { V2 } & :=\text { VehicleUD } & \text { map } & \text { IVE2E }
\end{array}
$$

where $F E 1 E$ changes in every action which starts with $V E$ the $V$ to 1 . The function $f V E 2 E$ is analogous. The unidirectional ring with two vehicles is defined as

$$
\text { Ring UD2 : }=(B C 0 \mathrm{wV}|\mathrm{BCl} \mathrm{wV}| \mathrm{BC2}|\mathrm{V} 1| \mathrm{V} 2) \text { allowonly } A_{\mathrm{XE}}
$$

Again we have RingUD2 $\approx$ SpecUD2. We report on statistics and on our experience using PVE for verification in detail in Section 5 .

\subsection{Refinement for allowing changes of direction}

We are now turning towards a design which allows vehicles to change their direction of movement. Opposed to the unidirectional case the notions predecessor and successor are no longer static. For an unambiguous 
reference to the neighbours of a block we use the notions left and right below. We assume the blocks of the track to be numbered in a linear order. We call the neighbour with a lower (higher) block number the left (right) neighbour. This definition can be extended easily for cyclic tracks (with at least 2 blocks). We will call the direction from left to right positive and the opposite direction negative.

Specification The specification of a ring with three blocks and one or two vehicles does not get much more complicated for the bidirectional case, see Fig. $9($ a) for the transition system SpecBD1 and Fig. 9(b) for the transition system SpecBD2.

(a)

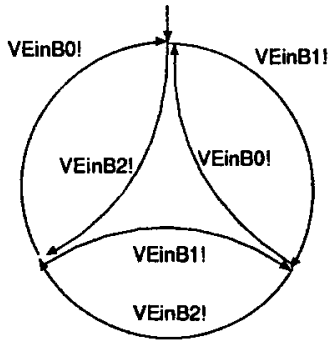

(b)

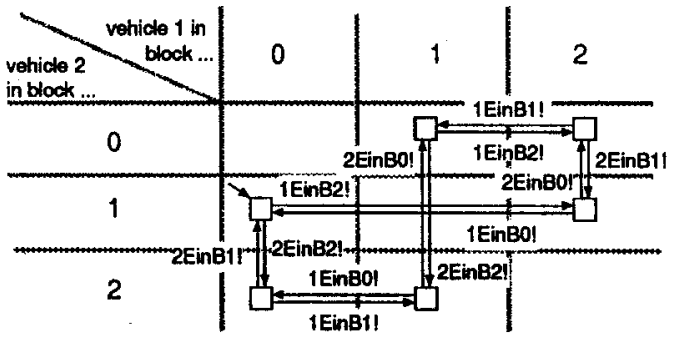

Figure 9: Specification of a bidirectional ring with three blocks and one (a) or two (b) vehicle(s)

The assumptions on the vehicle however are nore involved as we allow the vehicle to change its direction of movement at any time. The transition system VehicleBD $i$ for a vehicle which moves through a ring of three blocks starting in block $i$ is illustrated in Fig. 10. Observe that the outer (inner) circle corresponds to positive (negative) direction of movement and that the vehicle is allowed to perform a change of direction at any time. Only when the vehicle's reference point crosses a block boundary (which is assumed to be an atomic transition) a change of direction is not allowed between the notifications of the corresponding block controls and of the external observer.

Duplication of states In process algebra the labels of transitions are static, consequently it is impossible to modify the previously used action patterns dynamically depending on the current direction. As a remedy we duplicate each process in the block control. One copy is modified for positive direction (predecessor=left, successor $=$ right), the other copy is rewritten for negative direction.

Of course at each moment only one direction is valid in a block and consequently only one copy of a control process is allowed to be active (i.e. to perform actions). For the internal control process of a block control this is achieved by joining the initial states of the copies (cf. Fig. 12). We could do the same with the predecessor and successor control processes. Ilowever, if a vehicle changes its direction a reservation of a neighbour block will turn into a reservation for this neighbour. Therefore, we prefer to join the initial stales of the predecessor control for positive direction and of the successor control for negative direction to form a new process which is called left neighbour control process (NCL-process). Symmetrically a right neighbour control process (NCR-process) is formed. The shaded parts in Fig. 12 indicate those parts of the NCL- and NCR-process which result from this join.

Additional transitions As may be seen from Fig. 12 there are a number of additional transitions within the three processes of a complete block control. Their need is best explained with Fig. 11. The figure shows ten significant positions of a velicle, they are named by circled numbers. As in the unidirectional case for each position there is a corresponding state of the block control which is in turn a triple of the states of its components (cf. Fig. 12 for state names). However, as the vehicle may move in both directions for each position there are two triples. The pos-triple and the neg-triple correspond to positive and negative direction respectively.

The change from one position to the next usually involves several transitions of the block controls which are occupied by the vehicle. In Fig. 11 we only show one significant action. Ilowever, a few changes of position involve no transition of the block controls, the corresponding arrows are unlabeled. Solid (dashed) arrows correspond to positive (negative) direction of the vehicle.

Note that a vehicle is in position 1 with respect to a specific block if and only if it is in position 6 with respect to its left neighbour block. Similar facts hold for positions $2 / 7,3 / 8,4 / 9$ and $5 / 0$. 


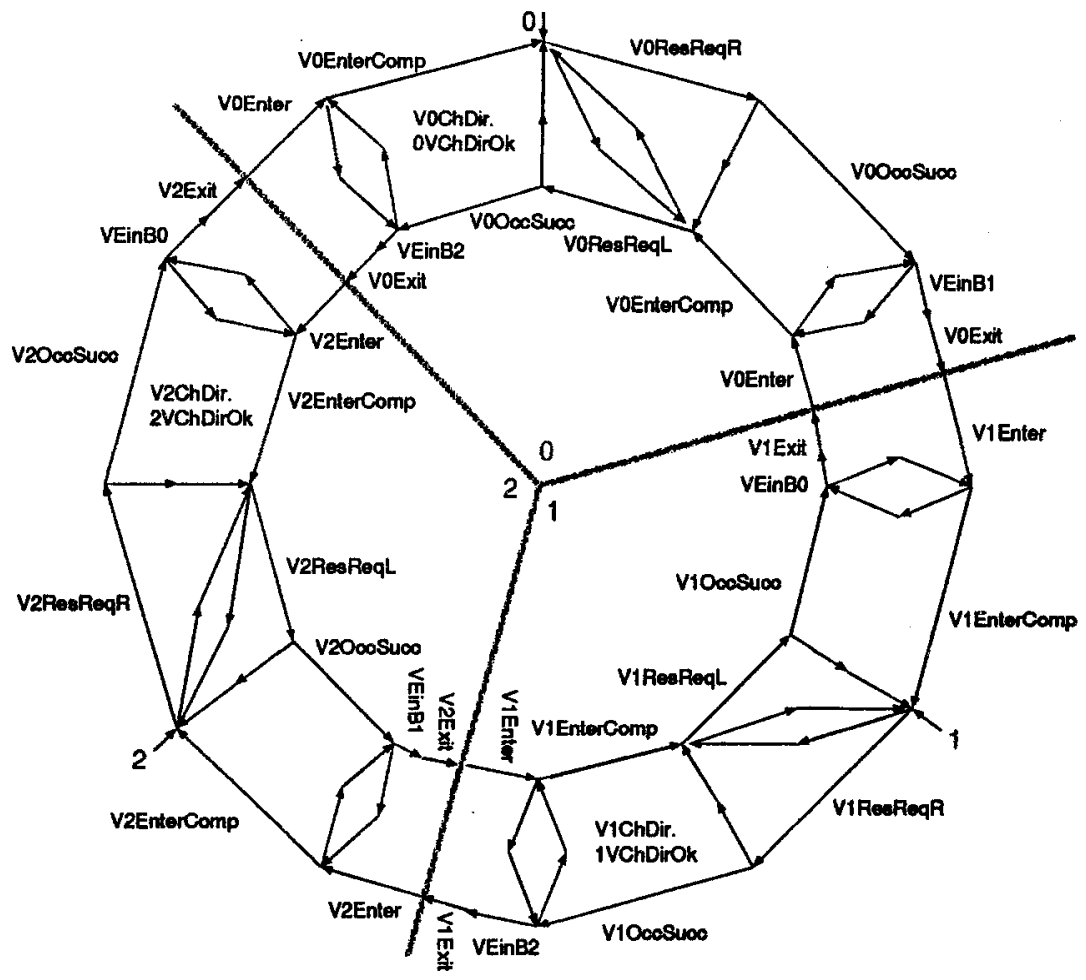

Figure 10: $\mathrm{A}$ bidirectional vehicle moving in a ring of three blocks

Fig. 11 shows which state clianges within a block control are expected when a vehicle changes its position. The block control shown in Fig. 12 is enhanced by additional transitions in order to ensure these changes of state.

To explain these transitions let us look at a vehicle moving in positive direction which is in position 5 with respect to its controlling block $A$. This means that the vehicle is in position 0 with respect to $A$ 's right neighbour block $B$. A change in this situation is very simple, it just means that the IC-process of $A$ has to change from IC3P to IC3N. Confer Fig. 12 to see how this is handled.

If the vehicle runs out of space as it approaches a block boundary it changes to position 6 and to position 1 respectively. Comparing the pos-triples shows that block control $A$ has to change its NCR-process from NCR1 to NCR3 and block control $B$ has to change its IC-process from IC1 to IC2P and its NCL-process from NCL1 to NCL2. It becomes clear that these three transitions are exactly what the construction in Fig. 7 does using the actions $A B R e s R e q, B B R e s e r v e L$ and BAResOk.

Let us now turn to a change of direction. Assume a vehicle which has reached position 6 and position 1 respectively decides to change its direction of movement. A change of direction in this situation implies a change of position from 6 to 5 and from 1 to 0 . Checking the differences between the pos-triple for position 6 and the neg-triple for position 5 as well as those between the corresponding triples for positions 1 and 0 one gets all state changes which have to be performed. In $A$ the IC-process has to perform the transition from IC3P to IC3N, thus turning the direction, and the NCR-process has to change from NCR3 to NCR1, thus cancelling the reservation of block $B$. During this change from NCR3 to NCR1 the NCR-process issues the IRRelReq-signal to the block control $B$. To prevent a new reservation process to start before the release operation is completed, the NCR-process waits for an acknowledgement called RIRelOk. The state NCL2 of $B$ 's NCL-process indicates that the left neighbour (which is $A$ in this case) has a reservation for block $B$. If a release request comes in, the process notifies its internal control process via URelease. The IC-process of $B$ completes the cancellation with $I L R$ RelOk which the NCR-process of block $A$ is waiting for. 


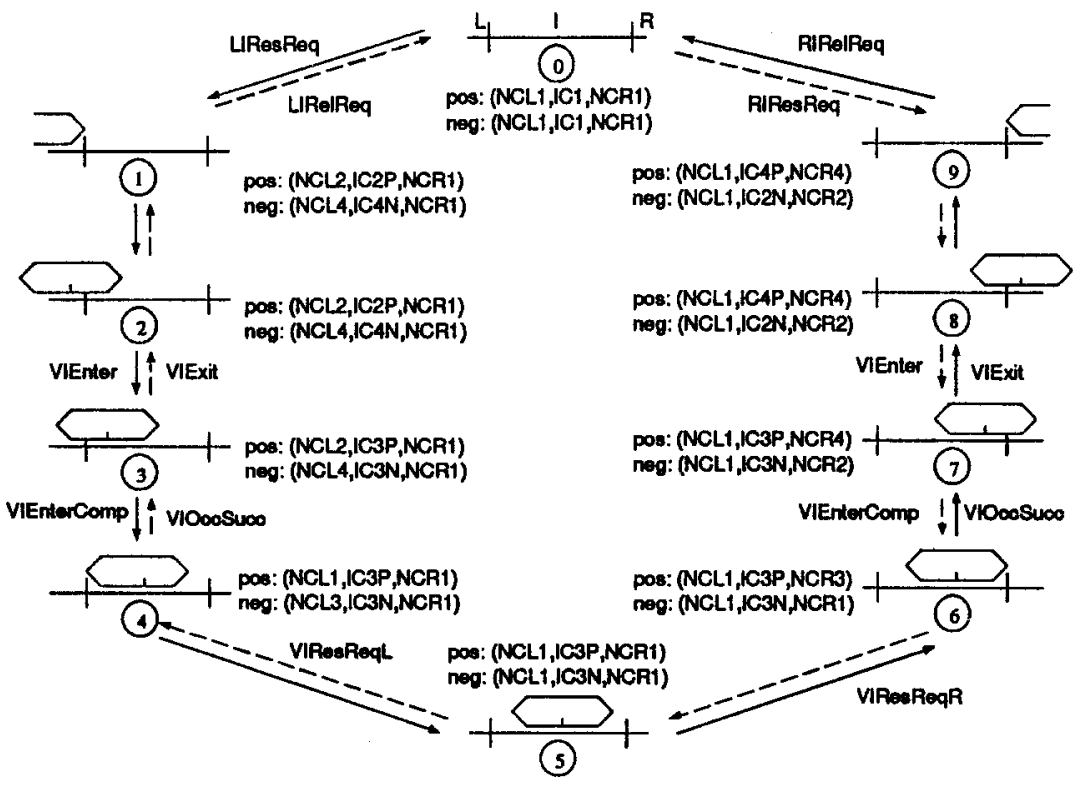

Figure 11: A vehicle passes through a block

Note that it is essential for absence of deadlocks in our design that the IC-process of $B$ comes back to its inital state after the NCL-process did. In fact we have made the error to program the synchronizations in the wrong order. This may cause a deadlock if a new reservation starts before the release operation is completed.

In a similar way the change of direction of a vehicle which is in position 7 with respect to its controlling block $A$ and in position 2 with respect to its right neighbour $B$ is handled. In this case no change of position is implied. An exiting vehicle becomes an entering one and vice versa. Consequently a reservation of a neighbour block turns into a reservation for that block. Here it becomes clear why we decided to join an SC-process with a PC-process. We use the new action IRDirExit to notify $B$ that the vehicle has switched its direction and hence is now exitiug $B$. In a symmetrical manner we use the action IRDirEnter for a cliange from negative to positive direction in the same positions.

The full model and its verification So far our design has become quite complicated and again we wish to ensure the correct function. The block control which is shown in Fig. 12 is defined by the following process algebra term.

$$
\text { BlockControlBD :=(NCLI } \mid \text { ICI } \mid \text { NCR1 }) \backslash A_{I I}
$$

Again $A_{I I}$ denotes the set of actions starting with $I I$. Similarly a block control which already controls a vehicle with positive direction of movement is written as

$$
\text { BlockControlBDWith Veh := }(\text { NCL1 } \mid \text { IC3P } \mid \text { NCR1 }) \backslash A_{I I} \text {. }
$$

Processing this in PVE results in two transition systems each with 6867 states. This seems surprising since during our construction we had very few combinations of triples of stales in mind (cf. Fig. 11). The reason for the large state space is that we have not made full use of the fact that communication between a block control and a vehicle occurs only if the velicle is controlled by the block control. For example each NC-process is able to accept a VIResReq $X$-signal from a vehiclế even if there is no vehicle in the block, i.e. even if the internal conirol is not in state IC3X.

The full system with one vehicle consists of three copies of the block control and the vehicle process shown in Fig. 10. It is given by the following definitions. 

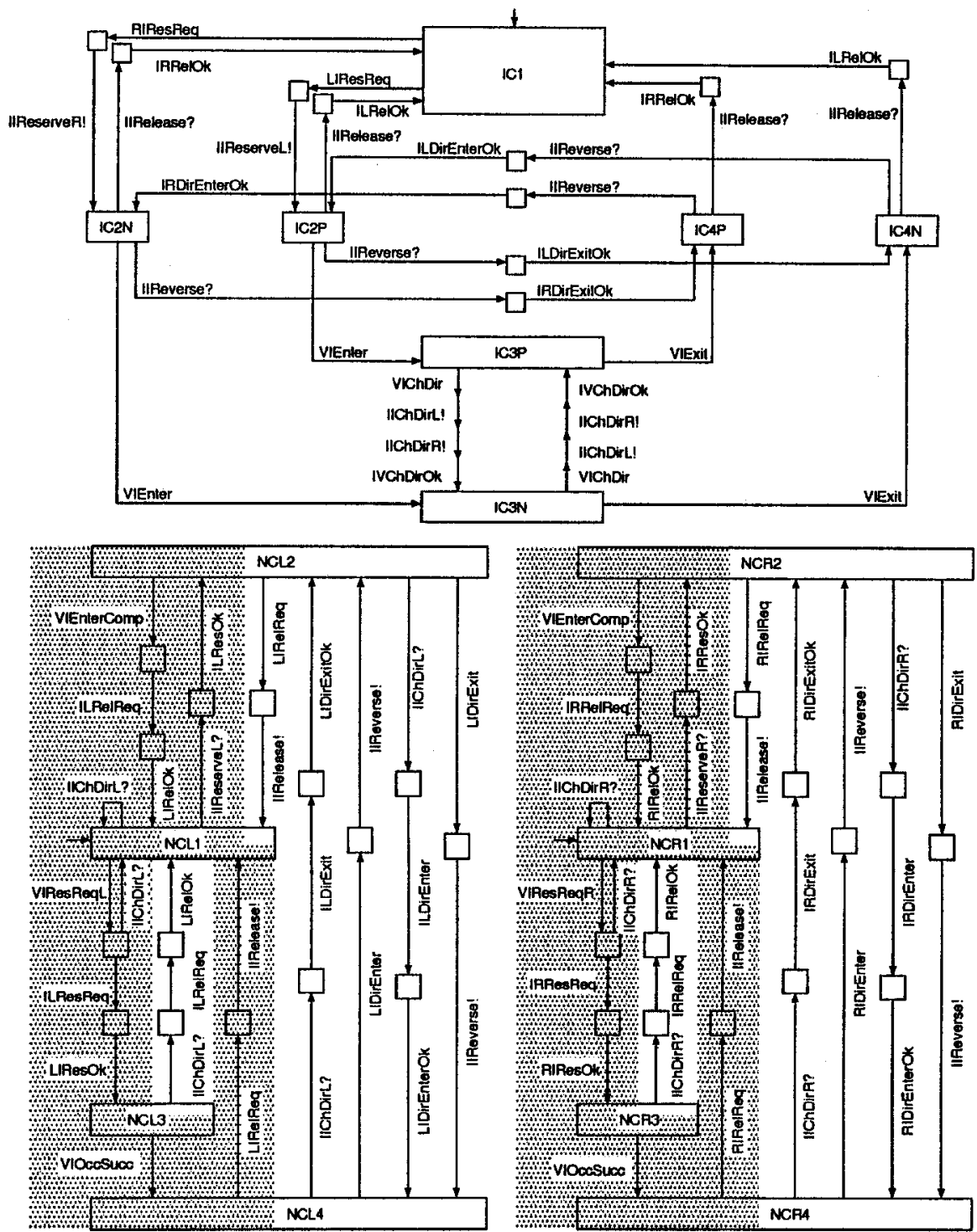

Figure 12: The block control for bidirectional vehicles 


$$
\begin{aligned}
& B C 0 w V:=\text { BlockControlBDWithVeh map fLIR201 } \\
& B C 1:=\text { BlockControlBD map ILIR012 } \\
& B C 2 \quad:=\text { BlockControlBD map fLIR120 } \\
& \text { RingBD1 :=(BCOwV } \left.|B C 1| B C 2 \mid \text { Vehicle } B D_{0}\right) \text { allowonly } A_{V E}
\end{aligned}
$$

Here the function $f L I R 201$ maps the lethers $L, I$ and $R$ in the first and second position of action names to 2,0 and 1 respectively. The set $A V E$ contains all actions which start with $V E$. $A$ quick PVE-proof shows that RingBD1 $\approx$ SpecBD1.

The same track carrying two vehicles is defined as follows.

$$
\text { RingBD2 : }=(B C 0 w V|B C 1 w V| B C 2|V 1| V 2) \text { allowonly } A_{X E}
$$

The processes $B C 1 w V, V 1$, and $V 2$ are understood in a similar way as in the previous section. Again we have $\operatorname{Ring} B D 2 \approx S$ secBD2. Detailed statistics of the verification are reported in the following section.

Having reached the final solution it may be observed that the coupling of the three components within the block control is rather tight. Indeed it is possible to give a solution for the block control with just one component which has less than twenty states. However, the splitting into three components is motivated by a natural functional decomposition, furthermore in practice the components serve for additional tasks which should be separated correspondingly.

\section{Verification in PVE}

The main results of the verification were already described above. This section shows how PVE was used as a development tool along the whole process of refining the design. In addition, we give verification results on the mutual exclusion requirement which were skipped above. Some statistics on the performance of PVE prove that we are able to handle real life systems without hampering the design process.

Using PVE for supporting the design process Developing the unidirectional version of the block control turned out to be straightforward from an earlier draft design and was easy to be proved correct.

A first, naive attempt to add changes of direction showed us that further development requires some method of "small development steps". We decided to make the development along a series of increasingly more complex vehicles. While the easiest vehicle does not perform changes of direction if any neighbour block is involved, the most complex vehicle (cf. Fig.10) performs changes of direction arbitrarily. After each step of improvement thè system RingBD1 was checked against SpecBD1 for equivalence. The time for generating the system never exceeded a minute while equivalence checking for systems containing still some errors was very likely to take up to 30 minutes. Consequently it is very important to have a fast tool even for relatively small systems. During this case study almost every incorrect intermediate development of the block control had some deadlocking behaviour. On the other hand the mutual exclusion requirement turned out to be very stable with respect to modifications. Hence we concentrated our raid for errors on deadlocks. For practical use it is essential to get quick answers on questions like "ls there any $\tau$-cycle which cannot be left by a visible action?" Our model checker takes no more than a few seconds for that (see the statistics below).

In the course of the refinement process the complexity of the errors found was increasing. One of them took a minimum of three changes of direction and a full run through the ring to get block controls confused. Later, when a second vehicle was added the length of pathes to erroneous behaviour grew even further. We believe that there is no possibility for detection of errors like these without a tool like PVE.

Additional verification results To check the main safety property, i.e. the mutual exclusion of vehicles in blocks, we send a pair of special test vehicles along the track. In every state they indicate the number(s) of the occupied blocks. Fig. 13 shows a fragment of a vehicle which corresponds to a run through a block in positive direction. The complete transition system (like Fig. 10) consists of six copies of this fragment. Fig. 13 shows only one transition for a change of direction, the others are to be added canonically. Using the action $V E$ EccBI vehicle $V$ indicates to an external observer that it is currently occupying block number $I$. The property "two vehicles never occupy the same block" is formalized in the following formula $\Gamma$ of the $\mu$-calculus:

$$
\begin{aligned}
& \nu X .(\neg(\langle 0 \text { EoccB0 }) \text { true } \wedge\langle(1 \text { EoccB0 }) \text { true }) \wedge \\
& \neg(\langle 0 E \text { occ } B 1\rangle \text { true } \wedge(1 \text { Eocc } B 1\rangle \text { true }) \wedge \\
& \neg(\langle 0 E \text { occ } B 2\rangle \text { true } \wedge(1 E \text { occ } B 2\rangle \text { true }) \wedge[.] X)
\end{aligned}
$$

The last column of Table 2 shows how long it takes to check this property for the three-block ring. 


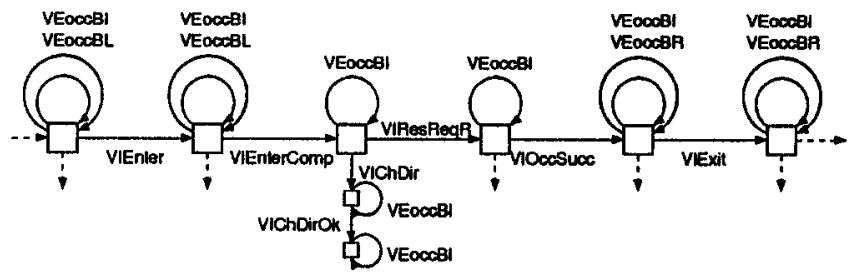

Figure 13: Verifying mutual exclusion of occupation of blocks

Statistics Below we present some statistics on the performance of PVE. They show that the tool's performance is feasible for the use in a stepwise refinement process. Since most time and space consuming problems occur when analyzing systems which contain errors (which are not listed here), performance must still be improved. All results were obtained on a Sun SPARCstation 2.

Table 1 summarizes results for the unidirectional version.

\begin{tabular}{r|r|r|r|r|} 
& \multicolumn{3}{|c|}{ generating } & $\begin{array}{c}\text { checking } \\
\text { transition system }\end{array}$ \\
& $\ldots \approx$ SpecUD $_{\mathbf{i}}$ \\
\hline & \#states & \#trans & time & \\
\hline BlockControlUD & 180 & 425 & $\ll 1 \mathrm{~s}$ & \\
\hline RingUD1 & 180 & 425 & $\ll 1 \mathrm{~s}$ & $8 \mathrm{~s}$ \\
\hline RingUD2 & 180 & 306 & $\ll 1 \mathrm{~s}$ & $5 \mathrm{~s}$ \\
\hline
\end{tabular}

Table 1: Stalistics for unidirectional version

The results for the bidirectional version are more interesting. 'The line for minBlockControlBD shows the size of the minimized version of the block control. It takes $80 \mathrm{~s}$ to compute it from BlockControlBD. The line for RingBD2* refers to the system which contains two test vehicles as described above for checking the formula $r$.

\begin{tabular}{r|r|r|r|l|l|} 
& \multicolumn{3}{|c|}{ generating } & \multirow{2}{*}{$\begin{array}{c}\text { checking } \\
\text { transition system }\end{array}$} & $\begin{array}{c}\text { checking } \\
\text { formula } \Gamma\end{array}$ \\
\hline & \#states & \#trans & time & & \\
\hline BlockControlBD & 6867 & 19138 & $17 \mathrm{~s}$ & & \\
\hline minBlockControlBD & 4266 & 17528 & & & \\
\hline RingBD1 & 5004 & 12342 & $26 \mathrm{~s}$ & $38 \mathrm{~s}$ & \\
\hline RingBD2 & 17280 & 48624 & $143 \mathrm{~s}$ & $13 \mathrm{~s}$ & \\
\hline RingBD2* & 16320 & 56880 & $140 \mathrm{~s}$ & & $29 \mathrm{~s}$
\end{tabular}

Table 2: Statistics for bidirectional version

\section{Conclusion and future work}

In this case study we have investigated the use of process algebra techniques for the design and verification of the distributed control of track vehicles. We have made a stepwise refinement and at each step the design has been verified using the automatic lool support PVE. We found several errors during our design. We claim that we have been able to locate these errors only due to the support given by PVE. This case study is industrially motivated. It concerns a certain aspect of a control problem only. However this aspect is trealed in full depth on the design level. We see this case study as evidence that a formal verification of parallel processes with process algebra techuiques is feasible if state-of-the-art tool support is available.

Ilowever using process algebra and PVE also indicaled where further developments are needed. Firstly process algebra should be enlianced by a more user-friendly facility to express communication links. Several errors resulted from typos concerning actions. In addition, the limitation to a static use of actions made the bidirectional solution more complex. It would be interesting to see if e.g. the $\pi$-calculus [15] would ease the formulation of the solution. Secondly the debugging aid of PVE has to be made more powerful. 
It should be noted that the verification approach in this paper verifies the correctness of particular topologies of the track with particular numbers of vehicles. It would be desirable to prove for the design of the block control that it belaves correclly in arbitrary topologies (e.g. in rings of arbitrary length). Future work will investigate suitable verification approaches.

Acknowledgement We thank our collegue Knigge for his cooperation. We also acknowledge joint work with Hans-Albert Schneider, Thomas Filkorn, and Reinhard Enders. Two anonymous referees have supplied helpful comments.

\section{References}

[1] J. Baeten and W. Weijland. Process Algebra, volume 18 of Cambridge Tracts in Theoretical Computer Science. Cambridge University Press, 1990.

[2] J. A. Bergstra and J. W. Klop. Algebra of communicaling processes with abstraction. Theoretical Compuler Science, 37:77-121, 1985.

[3] S. Brookes, C. Hoare, and A. Roscoe. A theory of communicating sequential processes. Journal of the $A C M, 31: 560-599,1984$.

[4] J. R. Burch, E. M. Clarke, K. L. McMillan, D. L. Dill, and L. J. FIwang. Symbolic model checking: $10^{20}$ stales and beyond. In Proceedings of the 5th IEEE Symposium on Logic in Computer Science, Philadelphia, pages 428-439, 1990.

[5] K. M. Chandy and J. Misra. Parallel Program Design: A Foundation. Addison-Wesley Publishing Company, 1988.

[6] R. Cleaveland, J. Parrow, and B. Steffen. The concurrency workbench. In J. Sifakis, editor, Automatic Verification Methods for Finite State Systems. Proceedings, Grenoble, 1989, volume 407 of Lecture Notes in Computer Science, pages 24-37, Berlin et al., 1990. Springer.

[7] R. Cleaveland and B. StcfTen. A linear-lime model-checking algorithm for the alternation-free modal mu-calculus. In Compuler Aided Verification. Proceedings, Aalborg 1991, pages 79-92, 1991.

[8] R. de Simone. Higher-level synchronizing devices in MEIJE-SCCS. Theoretical Computer Science, $37: 245-267,1985$.

[9] E. A. Emerson and C.-L. Lei. Efficient model checking in fragments of the propositional mu-calculus. In Proc. of the First Annual Symp. on Logic in Computer Science, pages 267-278. Computer Society Press, 1986.

[10] R. Enders, T. Filkorn, and D. Taubner. Generating BDDs for symbolic model checking in CCS. In K. G. Larsen and A. Skou, editors, Computer Aided Verification. Proceedings, Aalborg 1991, pages 263-278, 1991.

[11] K. Estenfeld, H.-A. Schneider, D. Taubner, and E. Tidén. Computer aided verification of parallel processes. In A. Pfitzmann and E. Raubold, editors, VIS '9l Verläßliche Informationssysteme. Proceedings, Darmstadt 1991, volume 271 of Informatik Fachberichte, pages 208-226, Berlin, 1991. Springer.

[12] P. Inverardi and C. Priami. Evaluation of tools for the analysis of communicating systems. EATCS Bulletin, 45:158-185, 1991.

[13] R. Milner. A Calculus of Communicating Systems, volume 92 of Lecture Notes in Computer Science. Springer, Berlin, 1980.

[14] R. Milner. Communication and Concurrency. Prentice Hall, New York, 1989.

[15] R. Milner, J. Parrow, and D. Walker. A calculus of mobile processes, Parts I and II. LFCS Report Series ECS-LFCS-89-85 and 86, LFCS University of Edinburgh, June 1989.

[16] S. Owicki and D. Gries. An axiomatic proof technique for parallel programs I. Acta Informatica, 6(1):319-340, 1976.

[17] R. Paige and R. E. Tarjan. Three partilion refinement algorithms. SIAM J. Compul., 16(6):973-989, 1987.

[18] W. Reisig. Petri Nets, volume 4 of EATCS Monographs on Theorelical Computer Science. SpringerVerlag, Berlin, 1985.

[19] C. Stirling and D. Walker. Local model checking in the modal mu-calculus. In J. Diaz and F. Orejas, editors, TAPSOFT '89. Volume 1., Proceedings, Barcelona 1989, volume 351 of Lecture Notes in Computer Science, pages 369-383, Berlin, 1989. Springer.

[20] D. Taubner. Finite Representations of CCS and TCSP Programs by Automata and Petri Nets, volume 369 of Lecture Notes in Computer Science. Springer, Berlin, 1989. 